\title{
Summaries of Discussions at 50th Anniversary ORCA Symposium
}

\section{Defining and Diagnosing Caries \\ Chairs: B. Angmar-Månsson, A.S. Lussi}

Several presenters pointed out the importance of early detection of caries lesions, which would improve the possibility of successful preventive intervention. However, early detection of the signs of caries should never trigger early operative intervention. In the future, early detection should take into account molecular and chemical aspects of the process.

Meaningful research should not aim at repetition of known facts but should implement knowledge in new designs of studies. These studies will be more complex in the future, in line with the multifactorial nature of caries and the skewed distribution of the disease in the population, with a relatively small number of individuals having a large caries experience.

A preventive programme has to be adapted to the society where it is supposed to work; no programmes can be used in all societies. Further, it is very difficult, if not impossible, to implement preventive programmes as long as politicians and patients consider caries not to be a problem. As long as these groups hold this belief, they will ignore preventive programmes. Preventive programmes can only be successful if behavioural scientists are involved.

\author{
How to Prevent Caries? Role of the Biofilm \\ Chairs: D. Beighton, C.H. Sissons
}

Three main topics were discussed. The responses from the speakers and from the floor underlined a lack of commonality of views.

Caries is not an infectious disease but a dysfunction of the normal microflora - there are no caries pathogens. Sissons agreed that oral bacteria were functioning 'normally', although perhaps in a different environment, but their proportions may have changed. Marsh stated that plaque is variable but all sites in all people, even disease-free individuals, harbour most bacteria, including those described as 'pathogenic'. Changes in the oral environment, e.g. sugar, inflammation, smoking, drugs affecting saliva, lead to an increase in 'pathogens' caused by ecological imbalances. Guggenheim agreed. Since 1967, caries microbiology had been dominated by Streptococcus mutans research, but $S$. mutans infection was opportunistic, as explained by the ecological plaque hypothesis. While $S$. mutans had an important role in caries, it was not the only cariogenic organism. Twetman thought that this implied that we should not throw away chlorhexidine, to which people respond differently with respect to $S$. mutans numbers. There was no direct association of mutans streptococci with caries. Russell thought more work was needed on host/bacteria interactions, but it was a sound principle to keep approaches simple.

\begin{tabular}{ll}
\hline KARGER & ( ) 2004 S. Karger AG, Basel \\
0008-6568/04/0383-0325\$21.00/0 \\
$\begin{array}{l}\text { Fax +4161306 12 34 } \\
\begin{array}{l}\text { E-Mail karger@karger.ch } \\
\text { www.karger.com }\end{array}\end{array}$ & $\begin{array}{l}\text { Accessible online at: } \\
\text { www.karger.com/cre }\end{array}$
\end{tabular}


Summary: There was a degree of unanimity amongst the panel members, but the audience was not as united, especially as to the role of S. mutans.

S. mutans has a minor role in caries. W.H. Bowen emphasised the importance of pellicle as a conditioning film that determines initial adherence and contains many enzymes, in particular glucosyl transferases (GTF). Because the major polymer in plaque is $\alpha-1,3$-glucan, which also binds GTF, an important role of mutans streptococci is to provide the glucan responsible for this stickiness. A decrease in GTF leads to a caries reduction in rats, suggesting both the importance of $S$. mutans and a need to focus on experiments to decrease virulence factors. $\mathrm{He}$ also pointed out that antimicrobial agents are not effective against caries. Marsh agreed that pellicle and plaque matrix need more research. Guggenheim thought that there was a question as to whether glucan-induced adhesion is specific or non-specific. Russell said results in rats on immunization against AgI/II showed that GTF has a role in caries. When asked why, if vaccines are successful, the normal immune system cannot control mutans streptococci, he replied that there is evidence for the production of antibody to plaque $S$. mutans, but that we do not know the level of mucosal immunity required to give protection. Organisms also adversely direct immune responses for their own benefit.

Summary: It has been clearly demonstrated that immunization against 'S. mutans caries' in rats works, but these experiments have yet to be replicated in humans.

Only the study of plaque biofilms is relevant to in vivo processes. C. Robinson said in his group's in vivo system $\mathrm{F}$ does not completely penetrate into plaque and commented that in the analysis of biofilm structure there can be problems with limited stain uptake and masking by EPS. Guggenheim replied that staining was not a problem in his group's model. S. Petti pointed out that there is a need to focus on the floras associated with different types of caries - root, enamel and dentine. $C$. Splieth asked why studies seem to be confined to only five species: why not use pooled plaque? Guggenheim stated that standardization would be lost, and a reproducible procedure is needed for testing antimicrobials (referring to his in vitro model).

Summary: Methods need to be developed to study human plaque in situ, to fully understand the complex microbial-host interactions required for the development of oral diseases.

\section{How to Prevent Caries? Role of Saliva}

\author{
Chairs: J. Tenovuo, L. Tabak
}

It was agreed that caries is a 'complex disease', influenced by host and microbial genes, infectious agents, diet/nutrition, environment, behaviour and societal factors. The oral milieu is dominated by a complex fluid saliva - which contains many molecules that interact with each other, with micro-organisms and with host cells. All these interactions differ somewhat between individuals and to get a better picture of their role in the caries process the scale of analysis needs to be increased, using genomic, proteomic and other new molecular approaches to interrogate all salivary proteins simultaneously. This should be done in real time to identify all relevant components at various stages of the disease, perhaps exploiting advances in biosensors.

It was agreed that no single salivary protein or component was decisive in initiation and progression of caries. Mucins and water were believed to be important, but interactions with other protective proteins and agents must also be considered. These events were very difficult to study in vivo because no patients (except those with selective IgA deficiency) were known to lack only one protein. Clearly salivary antimicrobials were present at insufficient concentrations to prevent dental caries totally and might be more important in keeping non-oral bacteria away than in regulating the oral microflora. One reason for the many problems associated with dry mouth was likely to be the reduced output of all protective salivary factors in severe hyposalivation. Reduced clearance of carbohydrates and acids likely contributed as well. Minor salivary glands, with their rich content of protective proteins, were very important in creating healthy local environments.

\section{How to Prevent Caries? Role of Fluoride}

\section{Chairs: J.D.B. Featherstone, L.C. Chow}

The key points made by the speakers during the discussion period are summarized here.

\section{ten Cate}

Availability of more effective caries-preventive products. This often depends more on corporate marketing decisions than on scientific or technological advances. A major manufacturer once stated that it is less costly and 
more profitable to launch a new toothpaste that claims improved whitening than one that claims superior anticaries effects.

Mechanisms of fluoride action. A usually neglected fact is that dental mineral is a defective carbonated hydroxyapatite that is much more acid soluble than hydroxyapatite, which in turn is more soluble than fluorapatite. During remineralization, the crystal surfaces become closer to fluorapatite, and are hence much less soluble than the original mineral. The true importance of any antibacterial action of fluoride needs to be tested clinically in a cariogenic environment.

Why a fluoride product may be less effective on certain individuals. The ability of a product/treatment to deliver sufficient fluoride to sites that are most vulnerable to caries, especially where there is a high bacterial challenge, should be given more attention in future research.

Dose response to fluoride in toothpastes and other products. The concept of dose response, as it applies to fluoride products, may need a better definition. The efficacy of delivery rather than concentration of fluoride in the product may be the key to success. The concept of slow-release fluoride has not been put into practice, but should be.

\section{Hellwig}

The primary caries-preventive mode of action of fluoride is post-eruptive. The evidence strongly supports this conclusion, but there is also evidence for some pre-eruptive effect and discussants insisted that we do not lose sight of a systematic effect.

What is the optimum fluoride concentration for topical treatments? There is ample evidence that topical fluoride affects lesion progress. However, fluoride concentration and retention in the oral environment rather than fluoride concentration in the product should be given more attention. The optimum for individuals will depend on the demineralization challenge.

\section{Hausen}

There are no novel fluoride measures in sight with an efficacy substantially higher than that of the preventives in general use today. Recent studies showed that oral fluoride retention from mouth rinse can be increased significantly without increasing the fluoride concentration in the rinse. A recent clinical study showed that simply controlling increased daily use of fluoride toothpaste reduced caries. These examples suggest that significantly more effective and practical fluoride products can be formulated. Discussants considered there is considerable room for improved fluoride delivery methods.

\section{Robinson}

On the effects of fluoride and magnesium on tooth mineral formation. A study on enamel mineral in growing rat incisors showed that a higher serum fluoride level led to a lower acid phosphate content, while a higher serum magnesium level led to a higher acid phosphate content.

On the effects of fluoride on cells and tooth development. Studies on tooth-forming cells in contact with fluoride at realistic micromolar concentrations are needed.

\section{How to Prevent Caries? Role of Sugars and Diet}

\section{Chairs: W.H. Bowen, P. Lingström}

Although sucrose is recognized as an important factor in the aetiology and pathogenesis of dental caries, it was pointed out that sucrose is rarely consumed alone and that attention should be paid to the diverse effects of food combinations. For example, a sucrose/maize mixture causes plaque $\mathrm{pH}$ to fall, whereas a milk/maize/sugar mixture raises it to alkaline levels. Inefficient swallowing might delay carbohydrate clearance from the mouth, and thus enhance the cariogenic potential of foods.

The suggestion was made that we no longer need to recommend restriction of sugar consumption as a means of preventing caries because of the advent of fluoride in various forms. In response, it was pointed out that inappropriate use of sugar was a public health problem. Caries was now strongly associated with poverty and even a small reduction in sugar use showed benefits. The question was also raised whether, from a public health perspective, it would be beneficial to re-consider adding cariostatic agents to sugar.

Several questions concerned the use of non-sucrose sweeteners such as polyols. Whether xylitol was simply non-cariogenic or actually caries-preventive remained unresolved. It was stated that chewing gum containing polyols when used by the mentally handicapped had a beneficial cleansing effect. Infants whose mothers used xybitol harboured much fewer $S$. mutans than controls. Users of xylitol also had plaque that was not very adhesive and harboured fewer $S$. mutans. Populations of $S$. mutans increased when sucrose was used. It was questioned whether the preventive effect of xylitol might be greater in relation to root surface caries compared with enamel caries. Although topical application of sorbitol usually results in a small fall in plaque $\mathrm{pH}$, one speaker mentioned a patient who developed rampant caries from persistently 
using sorbitol-containing lozenges, and in whom plaque $\mathrm{pH}$ fell to 3.5 following topical sorbitol application. All sweeteners currently on sale are generally regarded as safe, but it was questioned whether there are sufficient data to consider them safe for use by children.

In relation to early childhood caries, it was stated that most caregivers are well aware of what constitutes poor feeding behaviours, but persist in these poor habits nevertheless. Obtaining compliance with good dietary advice was regarded as a universal problem. The complexity of the diet/caries relationship was addressed. The exact role of diet was thought difficult to assess and it was stressed that not only what is consumed, but also individual patterns of consumption should be taken into account.

\section{How to Manage Caries}

\section{Chairs: J.P. van Amerongen, B.H. Clarkson}

\section{Are we ready to move from operative to} non-operative/preventive treatment?

J. Hamilton asked whether treatment strategy should be modified when delivering preventive non-operative care to patients who, like $50 \%$ of the US public, do not attend the dentist regularly. Pitts replied that it was important to adapt the preventive message for irregular attenders. In England, for example, the National Health Service is developing clinical care pathways for evidencebased preventive care and specific pathways for irregular attenders will present recommendations to patients and discuss the limitations of restoring teeth without addressing the causes of the disease. S. Petti pointed out that in Italy, which has no dental public health and a high caries incidence, there was no time for prevention and all caries has to be treated by operative procedures. Pitts replied that it was vital to address the causes of caries, as the evidence shows the futility, cost and limited outcomes associated with repeat restorative care. Zero said that efforts had been made for a number of years to get the 'medical model' of practice (in contrast to 'surgical care') accepted in the USA. This designation suggests that caries is an infectious disease. Pitts replied that it was vital to recognise that caries is a multifactorial disease; in most patients, it is not caused by bacteria alone, diet alone, poor oral hygiene alone or lack of fluoride alone. It was therefore important to give a valid, balanced message to both public and colleagues and not emphasise only one factor. He suggested that, rather than just talk of the 'medical' model, perhaps the dentist should be called an oral health physician to provide a better contrast to the traditional dental 'surgeon' who 'treated' caries rather actively and irreversibly. C.M. de Almeida commented that the best way to interrupt the caries process (and allow salivary remineralization) might be to teach patients to remove plaque completely and/or remove it professionally. M. Maltz agreed that the only available option for health professionals to promote oral health was to move towards non-operative/preventive strategies. For at least 20 years, sufficient data have been available to show that operative procedures can only prevent further development of a cavitated lesion. They do not prevent occurrence of new lesions that are likely to develop when the disease is not under control. Effective control required interference with aetiological and determinant factors. Pitts agreed but emphasised the need to convince dentists who have yet to make the conversion. In some countries, most dentists have already changed and practice in this way. In others, despite the evidence, many teachers and dentists choose to manage caries in the traditional way. ORCA and others have a continuing job to provide best evidence and best practice.

\section{How 'clean' must a cavity be before restoration?}

M. Maltz commented that asking whether or not to leave bacteria beneath a restoration overlooked the wellestablished consensus that conventional caries removal normally leaves bacteria beneath restorations. However, after incomplete removal of carious dentine and tooth sealing, the number of residual bacteria was significantly reduced, and the dentine underneath the provisional restoration acquired the characteristics of an inactive caries lesion. An important question was whether there were any differences in the residual bacteria after conventional carious dentine removal and after incomplete carious dentine removal and tooth sealing. Kidd agreed. M.J. Noack thought it was not a problem that teaching with respect to caries excavation during the last decades had been in error, because dentists had not followed this teaching in any case. There was evidence that in most cases some carious dentine is left, especially at the dentine-enamel junction. Kidd agreed. Noack thought two questions might influence strategy: how should non-compliant patients, who do not control plaque properly, be dealt with? As dental restorations in general leak, is the proposal for a less invasive excavation approach applicable only to adhesively sealed restorations or can it also be used for conventionally cemented restorations? Kidd thought more research was needed. C.M. de Almeida thought that the 
main problem in pulp survival was not bacteria in dentine, given a perfect seal at the interface with the cavity wall, but bacteria that had already penetrated to the pulp, producing reversible or irreversible pulpitis. Often the production of sclerotic and tertiary dentine was defined as a pulpal defence mechanism, but should perhaps be regarded more as a sign of a degenerative process: the consequence of inflammation, resulting in fibrosis and calcification. J.P. van Amerongen asked about the teaching of the new approaches and especially the criteria for determining the extent of excavation at the lesion periphery. Kidd enumerated the following: discussion of the literature, particularly the work of Mertz-Fairhurst (which she considered should be repeated by another group); when removing demineralised dentine, the enamel-dentine junction should be made hard (but not stain-free); demineralised tissue over the pulp should be excavated to the level of firm dentine provided there was no likelihood of pulpal exposure; deep lesions in symptomless, vital teeth should be gently excavated; soft, demineralised dentine can remain where its removal might expose the pulp; a permanent restoration is placed; there was no re-entry; caries dyes were not used.

\section{The future role of a molecular approach to} pulp-dentinal regeneration

A. Sotirovska-Ivkovska asked how, when the pulp is exposed because of caries, reversible and irreversible pulpal changes can be distinguished. When should the pulp be stimulated for dentine bridge formation? Tziafas answered that in the presence of irreversible pulpal changes, tissue preservation was not possible. Under active caries, irritating factors from bacteria and tissue breakdown affect the dentinogenic unit at the dentinepulp interface for long periods and progressively destroy it, so therapeutic regulation of tissue healing, regardless of treatment modality, is not possible. C.M. de Almeida suggested that pulp-dentinal regeneration might depend more on levels of bacterial penetration and of pulpal inflammation than on a molecular approach. Tziafas disagreed; the most important determinant of vitality and function of the dentine-pulp complex was restoration of the dentinogenic unit at the pulp-dentine interface. Bacteria, other exogenous stimuli, including traditional thera- peutic modalities, and inflammatory changes adversely affect the healing capacity of the dentinogenic unit. As a result, irregular hard tissue of unpredictable defensive nature is deposited, but this cannot block exogenous destructive stimuli effectively. New molecular approaches were aimed at specifically controlling the restoration of the dentinogenic unit. Tertiary dentine was the most appropriate matrix to protect the pulp from secondary infection. $O$. Fejerskov, following up de Almeida's comment, pointed out that pulpal inflammation had been shown to attenuate the ability of the pulp to respond to BMP application, which in the non-inflamed pulp had resulted in new dentine formation. O. Fejerskov then asked about the role of the superficial necrotic zone subjacent to the $\mathrm{Ca}(\mathrm{OH})_{2}$ in the formation of new matrix, given that $\mathrm{Ca}(\mathrm{OH})_{2}$ containing TGF- $\beta 1$ induces dentine bridge formation. Tziafas answered that odontoblast-like cells differentiated and reparative dentine was formed in relation to this zone. Preliminary reports had shown that a network of bioactive molecules, including fibronectin and growth factors, was created at the surface of vital tissue. A necrotic zone was not formed under experimental conditions in relation to pre-set (inert) $\mathrm{Ca}(\mathrm{OH})_{2}$ and it was hypothesised that addition of TGF- $\beta 1$ substituted for the zone of tissue necrosis and the associated molecular network.

\section{Getting Research into Clinical Practice}

\section{Chair: N.B. Pitts}

It was agreed necessary to acquire data on all age groups, particularly adults. J. Brown felt that research by general practitioners was necessary to acquire generalisable data and to ensure that training on prevention was delivered to general practitioners. W.H. Bowen asked about procedures for liability and ethics approval of research conducted in general practice. Clarkson replied that all projects went through the usual Research Ethics Committee procedures and that liability was covered through the National Health Service Trusts in which the dentists worked. 